\title{
Asesoramiento psicopedagógico: propuesta de intervención para reforzar las funciones básicas en una adolescente con déficit cognitivo moderado y severo.
}

DOI: $\underline{\text { https://doi.org/10.33262/ap.v3i2.41 }}$

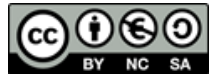

Psychopedagogical counseling: intervention proposal to reinforce basic functions in an adolescent with moderate and severe cognitive deficit.

Mónica Narciza López Pazmiño ${ }^{1}$ \& Luis René Indacochea Mendoza. ${ }^{2}$

\begin{abstract}
.
In Ecuador, the educational system works by means of norms and principles published by the Ministry of Education, which uses the updating and strengthening of the curricula, aiming at improving the quality of learning; From the institutional perspective, the educational units and centers select the activities proposed by the zonal districts in order to reinforce cognitive functions in a special way in boys and girls who present moderate and severe cognitive deficits. For the above, the study aims through psychopedagogical advice to consolidate the teaching process in the curriculum, didactics and pedagogy, guiding intelligence, thinking, language, memory, attention and understanding with a focus on intellectual functioning, adaptation and social skills. Vision that aims to reduce school dropout and low performance, raising self-esteem, self-worth, confidence and self-assurance, motivating the completion of tasks, increasing interest in learning. In collecting the information, it was decided to use the David Weschler (2005) test; In addition, a review of the academic performance achieved by the students during the first school semester was

\footnotetext{
${ }^{1}$ Afiliación Universidad Tecnológica Indoamérica, Facultad de Ciencias Humanas de la Educación y Desarrollo Social. Carrera Educación Básica y Educación Inicial, Ambato, Ecuador, email monilopez.2012@hotmail.es https://orcid.org/0000-0002-7537-6630

${ }^{2}$ Universidad Técnica de Ambato. Facultad de Ciencias Humanas y de la Educación. Carrera Psicopedagogía, Ambato, Ecuador, email luisrindacocheam@uta.edu.ec. https://orcid.org/0000-0002-0215-5263
} 
carried out. In the methodology, a correlational descriptive non-experimental approach has been used.

Keywords: Counseling, didactics, pedagogy, moderate and severe cognitive deficit, basic functions.

\section{Resumen.}

En el Ecuador el sistema educativo funciona mediante normas y principios difundidos por el Ministerio de Educación, que utiliza la actualización y fortalecimiento curricular, proyectando en mejorar la calidad en los aprendizajes; desde la perspectiva institucional, las unidades y centros educativos seleccionan las actividades propuestas por los distritos zonales con la finalidad de reforzar las funciones cognitivas de forma especial en niños y niñas que presentan déficit cognitivo moderado y severo. Objetivo. Analizar el asesoramiento psicopedagógico: propuesta de intervención para reforzar las funciones básicas en una adolescente con déficit cognitivo moderado y severo. Metodología. La metodología es cualitativa que tiene como finalidad profundizar en las características definiciones y teorías relacionadas con la problemática detectada además se aplica la indagación de campo y bibliográfica documental correspondiente a un diseño no experimental, el instrumento aplicado fue de (Folstein, Folstein, McHugh, \& Fanjaiang, 1975) que detecta trastornos cognitivos multifuncional, adaptado por (Lobo, Saz, \& Marcos, 2002) conformado por 35 preguntas (orientación, el tiempo, decodificación, atención, concentración, recuerdo, lenguaje y construcción visual. Se resalta que la prueba (WISC-R) valora la comprensión lectora, el razonamiento perceptual, la memoria de trabajo y velocidad de procesamiento. Resultados. Los rangos, población con conflictos intelectuales de discapacidad incluye, discapacidad leve (70 y 50), discapacidad moderada, con necesidad de supervisión y educación especial (50 y 35), discapacidad intelectual grave, o incapacitados (35 y 20), discapacidad intelectual profunda, daños neurológicos que limitan la actuación e interacción con el medio (menor a 20).Conclusión. El asesoramiento psicopedagógico consolida el proceso de enseñanza en el currículo, la didáctica y pedagogía, orientando en la inteligencia, el pensamiento, el lenguaje, la memoria, atención y entendimiento con un enfoque en el funcionamiento intelectual, la adaptación y las habilidades sociales. Visión que pretende disminuir el abandono escolar y el bajo desempeño, elevando la autoestima, la autovaloración, la confianza y seguridad en sí mismo, motivando al cumplimiento de tareas, incrementando el interés en el aprendizaje.

Palabras claves: Asesoramiento, didáctica, pedagogía, déficit cognitivo moderado y severo, funciones básicas.

\section{Introducción.}

La educación se ha constituido en un componente de justicia social, incluye la pluralidad de enfoques, temáticas y metodologías relacionadas con la calidad de formación y los resultados 
de aprendizaje. Los últimos tiempos en diferentes países de América Latina especialmente en Chile, Bolivia y Ecuador las innovaciones en el sistema educativo han fortalecido al proceso de enseñanza; lamentablemente la escasa participación del docente en eventos formativos ha limitado el manejo de planes de prevención que aporten en el desarrollo cognitivo moderado y severo; además la insuficiente utilización de técnicas y estrategias metodológicas ha perjudicado en la adquisición de las funciones básicas y su refuerzo.

Sistema educativo al estar orientado por la actualización y fortalecimiento curricular toma como eje la capacitación e innovación de los maestros y maestras siendo la meta alcanzar aprendizajes significativos. La insuficiente participación de los docentes y padres de familia en la labor pedagógica ha condicionado la afectividad, el conocimiento y apropiación de contenidos.

En varias instituciones educativas la limitada realización de intervenciones psicopedagógicas ha generado barreras en las adaptaciones curriculares de acuerdo a las necesidades particulares del estudiantado, perjudicando en el rendimiento académico y bienestar personal. Cabe resaltar el criterio de (Ausubel, 1983) el insuficiente cumplimiento de procesos que beneficien el desempeño docente desfavorece en la enseñanza de saberes (ser, hacer, conocer y vivir) factor que repercute en la resolución de problemas y compromiso ético.

En la institución educativa, el asesoramiento psicopedagógico es considerado efectivo en el proceso de enseñanza aprendizaje cuando el estudiante obtiene el conocimiento específico, explícito y representativo; Picco \& Orienti (2017) sostienen en el escrito titulado Didáctica y Currículo que el asesoramiento psicopedagógico es el procedimiento de ayuda y acompañamiento permanente a los sujetos en diversos aspectos para potenciar la prevención y el desarrollo humano con base en principios científicos y filosóficos. Mientras De La Oliva, Tobón, Sánchez, Romero, \& Posadas (2019) menciona: la orientación psicopedagógica tiene como propósito aportar a la formación de los seres humanos.

Se deduce que el asesoramiento psicopedagógico: orientación curricular, didáctica y metodológica es un proceso que contribuye en la obtención de información vocacional respecto a las aptitudes, intereses y expectativas del individuo; mediante la adquisición del conocimiento y principios teóricos favorece en la planificación, diseño, aplicación y evaluación de la intervención psicopedagógica preventiva, comprensiva, integral y continuada dirigida hacia los sujetos, las instituciones y el contexto comunitario, con el propósito de posibilitar, promover y contribuir en el desarrollo de los individuos durante las etapas de su vida, integrando a los diversos agentes educativos (orientadores, tutores, profesores, familia) y sociales.

El estudio permitirá mejorar los procesos de enseñanza para alcanzar un mejor rendimiento y desempeño académico. Partiendo de concepciones pedagógicas, didácticas, metodológicas, argumentativas y toma de decisiones aporta en el desarrollo cognitivo y social pretendiendo 
fortalecer la interrelación con la comunidad, el sistema de apoyo, los recursos y autonomía profesional; de esta manera disminuye las posibles barreras que limitan el proceso de la dinámica escolar e innovación en el aprendizaje cognoscente donde el docente asume la responsabilidad desde las corrientes interactivas, y reflexivas limitando la educación tradicional sustentado en el pensamiento práctico y teórico.

La indagación propone como objetivos: Diseñar un proyecto de asesoramiento psicopedagógico propuesta de intervención para reforzar las funciones básicas en estudiantes con déficit cognoscitivo moderado y severo. Revisar referencias actualizadas. Analizar las ventajas de la orientación psicopedagógica en el proceso cognitivo del alumnado.

\section{Asesoramiento Psicopedagógico}

La Psicopedagogía según Vásquez (2018), tiene el propósito de crear orientaciones que contribuyan en la formación del individuo, a través del análisis psicopedagógico de cada situación; Bertoldi, Enrico y Follari (2018) manifiestan que examina los aspectos de orden psicológico, formula métodos didácticos y pedagógicos eficientes; integra los procedimientos que abarcan la asimilación (nuevo conocimiento); acomodación (adaptación a la nueva información), centra su atención en modificar las estructuras cognitivas mediante la intervención.

Desde un marco conceptual García (2003), plantea el asesoramiento psicopedagógico a partir del diagnóstico, análisis e identificación de factores de riesgo en la institución y su contexto, abarca las necesidades en la organización de conceptos y el aprendizaje de la comunidad educativa; por tal razón utiliza la orientación curricular, didáctica y metodológica en la intervención, maneja estrategias e instrumentos, conllevando al progreso, la calidad y equidad.

Empleado el modelo Psicométrico, el cual es utilizado frecuentemente en las instituciones educativas a fin de clasificar a los alumnos con déficit cognitivo, de acuerdo con Jimenez Hernadez, citando a Grau y Bueno y Verdugo, (1.986), el deficiente mental en la puntuación de C.I. está una o dos sigmas por debajo de la media de la población, siendo inferior a 85 o 70 de C.I., según definiciones ha permitido una clasificación de los mismos en deficientes ligeros, moderados, severos y profundos (p. 14). Para el caso del estudio, se centra en el déficit cognoscitivo moderado y severo.

\section{Estudios sobre funciones básicas y discapacidad cognitiva}

Al incluir el desarrollo de las capacidades sensoriales e intelectuales orienta en el proceso dialéctico por tal razón las actividades de asimilación de conocimientos interioriza en las funciones superiores que fortalecen la toma de decisiones y facilitan la resolución de conflictos. Según García, Hernández y Peláez (2003) el asesoramiento psicopedagógico, la orientación curricular, didáctica y metodológica se relacionan con el conocimiento (fortifica 
la memoria, el lenguaje y la percepción). Por lo tanto la capacidad cognitiva se conecta con las habilidades del habla, entendimiento, reflexión, análisis y actuación. López, Sagñay, Vega y Mera (2019) siendo necesaria la coordinación de destrezas del autoconocimiento (empatía y relaciones interpersonales) de esta forma fortalece el pensamiento creativo, la autoestima y autonomía.

Según el (Instituto Colombiano de Bienestar Familiar (ICBF), 2010), la discapacidad cognitiva hace referencia a niños y niñas pueden presentar diferencias en su ritmo de aprendizaje debido a los factores biológicos sociales culturales; lo significativo es reconocer la existencia de particularidades que sustentan las diversidades individuales. El concepto a través del tiempo ha cambiado desde un enfoque individual hasta el social; además abarca diversos conceptos vinculados con el retraso mental, discapacidad intelectual. Por lo expuesto el autor da a conocer la siguiente terminología:

Las capacidades abarcan las habilidades sociales, participativas y comunicativas.

El contexto, son lugares donde los educandos aprenden juegan, socializan e interactúan por esta razón es necesario conocer las características del entorno en el cual se desenvuelven los niños con el propósito de adaptarlo a su edad, género y posibilidades intelectuales y sociales.

El funcionamiento vincula las capacidades con el entorno en un ambiente determinado, ofrece oportunidades de aprendizaje que benefician en la memoria, percepción, atención.

Según el (Instituto Colombiano de Bienestar Familiar (ICBF), 2010), la discapacidad cognitiva incluye las habilidades para desempeñarse en el ambiente cotidiano (bañarse, vestirse, comer) las experiencias sociales (expresión, diálogo, afecto, participación). Salud física y mental (nutrición, potencia visual y auditiva). El contexto (apoyo a la familia en los procesos de formación por la recreación inclusiva el respeto a las diferencias de aprendizaje.

Las causas de la discapacidad cognitiva se pueden ubicar dentro de los factores biomédicos relacionados con alteraciones genéticas o una inapropiada nutrición; incluye aspectos sociales vinculados con la interacción familiar como el rechazo a la carente protección parental; mecanismos conductuales (comportamientos inadecuados, actividades peligrosas abuso del alcohol y otras sustancias por parte de la madre); componentes educativos entre ellos la carencia de apoyo que limita el desarrollo intelectual. De acuerdo a lo expuesto se determina que la identificación de los orígenes de discapacidad cognitiva promueve una toma de decisiones de los padres, la familia, el cuidador, los maestros y el agente formativo.

\section{Déficit cognitivo moderado}

Como describe Manzano, \& Morocho (2018) el déficit cognitivo moderado, se manifiesta en un coeficiente intelectual entre 35-50, son considerados individuos que aún no aprenden a leer ni a escribir, su capacidad de aprender no le permite alcanzar un nivel de escolaridad mayor al correspondiente al segundo grado de primaria. Según Mañas, González, \& Cortés 
(2020), existen limitaciones en el aseo, alimentación y vestido, el correcto desempeño, necesita de ayuda. Dentro de las tareas formativas requieren supervisión, la mayoría participa en actividades sociales, tienen dificultad para reconocer los convencionalismos, pueden beneficiarse del entrenamiento en habilidades de autocuidado y hasta cierto punto laboral ( $\mathrm{p}$. 12)

Con el propósito de caracterizar el déficit cognitivo moderado de conformidad con lo indicado por (Rodríguez Yela, 2016, pág. 13), que emplea el DSM - 5 Novedades y criterios de diagnóstico propuesto por la American Association On Mental Retardin en el año 2013, expone lo siguiente:

Área conceptual, en la edad escolar, el progreso en lectura, escritura, matemáticas, la comprensión del tiempo y del dinero ocurre lentamente.

Área social, el individuo muestra una marcada diferencia con respecto a sus iguales en la conducta comunicativa.

Área práctica, el individuo puede cuidar sus necesidades personales como: comer, vestirse, higiene personal; aunque requiere de un periodo extenso de enseñanza y lleva tiempo que la persona pueda ser independiente, necesita de alguien que le recuerde.

\section{Déficit Cognitivo Severo}

El déficit cognitivo severo, denominado déficit cognitivo grave, según Manzano Sánchez \& Morocho Morocho (2018) se presenta en las personas con coeficiente intelectual situado entre $20-35$, muestran un desarrollo motor escaso, requieren cuidados casi totales, adquieren habilidades en tareas simples, contribuye parcialmente para su propio manteniendo un alto grado de supervisión (p. 12)

A fin de caracterizar el déficit cognitivo severo, según lo mencionado por (Rodríguez Yela, 2016, p. 13) emplea el DSM - 5 Novedades y criterios de diagnóstico propuesto por la American Association On Mental Retardin en el año 2013, indica lo siguiente:

Área conceptual, está limitada la consecución de habilidades conceptuales. El individuo tiene una escasa comprensión del lenguaje escrito o números, para cantidades, el tiempo y el dinero.

Área social, el lenguaje hablado es limitado en términos de vocabulario y gramática. El habla consiste en simples palabras o frases, puede ser complementada por estrategias aumentativas.

Área práctica, el individuo requiere el apoyo para efectuar las actividades de la vida diaria, incluyendo comida, vestido, baño y aseo; precisa supervisión todo el tiempo, no toma decisiones responsables sobre su bienestar o el de otros. 


\section{Formas de prevención}

Según Parra Gómez (2016) la discapacidad intelectual es una patología que afecta aproximadamente del 1 al 3\% de la población, su causa es diversa; sin embargo, el $47 \%$ de los casos tienen su origen en factores genéticos. La discapacidad intelectual integra parte del grupo de patologías difíciles de diagnosticar y tratar, provocando un impacto sustancial en la vida del individuo, su familia y la sociedad. (p. 6)

Las limitaciones cognitivas pueden pasar sin ser identificadas por parte de los médicos, e incluso en ciertas ocasiones por sus progenitores, quienes se dan cuenta cuando el niño ingresa a la escuela.

Parra Gómez (2016) considera que efectuarse dos evaluaciones sencillas que pueden servir de indicadores siendo estas:

- Balbuceo canónico: entre los cuatro a diez meses de edad el niño debe producir sonidos formados por lo menos con dos sílabas, una vocal y una consonante, articulándolas de manera rápida.

- Funciones pragmáticas: se estructuran alrededor del año de vida; es una expresión precoz de la estructuración del lenguaje en el niño.

En el análisis radican los siguientes puntos:

- Interacción social (registra al otro, llama la atención, saluda).

- Atención convocada (cautiva la atención de un adulto hacia un objeto que está percibiendo).

- Regulación conductual (lograr que otro ejecute una acción requerida por el) (p. 8)

La estimulación cognitiva se basa en diferentes actividades enfocadas en el mejoramiento de las capacidades mentales por esta razón se emplea principios de plasticidad mental que modifica las conexiones de cerebro; la estimulación cognitiva requiere de una intervención terapéutica que ayuda a retardar el deterioro que presentan las personas con estas características, evitando la desconexión del entorno, fortalece las relaciones sociales y potencia la autoestima; así se aporta en la memoria, expresión verbal, percepción visual y razonamiento requiriendo de tres aspectos principales: la paciencia, comprensión y flexibilidad ante cualquier tratamiento. Son un aporte los ejercicios de estimulación cognitiva mediante acciones lúdicas pueden ser juegos de carta, de mesa, crucigramas, sopas de letras entre otros.

Para prevenir los factores de riesgo biomédicos, sociales, conductuales y educativos se recomienda en el proceso de gestación recibir atención médica mediante controles periódicos; además realizar evaluaciones y exámenes clínicos con la finalidad de acoger orientaciones y recomendaciones; formalizar un seguimiento a las condiciones del niño o niña en el embarazo incluyendo los movimientos, crecimiento, el aumento de peso; leer o 
asistir a diferentes cursos, charlas o talleres donde el padre, la madre o cuidadores reciban la información relacionada con los cuidados; consumir alimentos que incluyan proteínas al igual que frutas y verduras; cumplir con la lactancia materna durante los primeros 6 meses de vida; efectuar acciones de acuerdo con las sugerencias médicas; no usar ningún tipo de droga ni alcohol o medicamentos no prescritos por los médicos.

Prevención durante el parto es oportuno la asistencia al hospital o centro médico para el nacimiento, la demora en recibir atención puede producir en el niño o niña perdida de oxígeno que trae como consecuencias un daño cerebral; además debe garantizar los entornos apropiados, es importante el cuidado por especialistas que respondan a las complicaciones que se presenten; desde esta perspectiva el alumbramiento en casa no garantiza las condiciones higiénicas y de salud adecuadas.

Entre las recomendaciones y cuidados especiales para el niño después de nacimiento (Instituto Colombiano de Bienestar Familiar (ICBF), 2010) menciona: asistir al especialista por lo menos una vez al mes; efectuar los exámenes solicitados que aporten en la verificación de su conducción física y mental; realizar el seguimiento a la talla y el peso; aplicar las vacunas de acuerdo a las indicaciones y orientaciones del médico dar la alimentación correspondiente a las necesidades de recién nacido; estimular el desarrollo, condiciones físicas y mentales; posibilitar un ambiente agradable y libre de peligros; por último brindar afecto y protección al infante.

Según (Instituto Colombiano de Bienestar Familiar (ICBF), 2010), para detectar la discapacidad cognitiva se requiere de exámenes especializados por ejemplo amniocentesis permite identificar alteraciones genéticas como el síndrome de Down, (toma de muestras de líquido amniótico de la madre con el propósito de observar cambios en las cédulas de los genes). La discapacidad, se puede divisar en el desarrollo lento que presenta el infante al caminar, el movimiento de la cabeza, dormir demasiado, ser tranquilo, su cuerpo es blando; al cambiar de pañal no se mueve a diferentes posiciones, demuestra desinterés por las personas y los juguetes, no crece ni aumenta de peso; por lo expuesto, se recomienda asistir a control y reportar las señales de alguna dificultad; buscar orientación con el programa de estimulación.

\section{Metodologia.}

El estudio presenta una metodología cualitativa incluye la revisión bibliográfica en la búsqueda, organización, síntesis y análisis desde la modalidad de campo, lo que facilita la adquisición de información e identificación de los principales autores que cultivan el asesoramiento psicopedagógico, las funciones básicas y el déficit cognitivo moderado y severo.

Es decir mediante la investigación de campo y bibliográfica documental se cumple con el objetivo de describir la importancia de las funciones básicas en el déficit cognitivo moderado 
y severo; además la indagación cualitativa y no experimental respalda a el escrito a través de la consulta en diferentes fuentes documentales, incluye libros electrónicos, artículos ensayos, opiniones, antologías expuestas en revistas especializadas.

El diseño de investigación fue no experimental porque no existe manipulación de variables por parte del investigador, empleándose en el sustento teórico información científica actualizada en referencia al desarrollo de las funciones básicas y déficit cognitivo, incluye teorías publicadas a nivel nacional e internacional. Entre los investigadores de mayor trascendencia sobresalen:

(Folstein, Folstein, McHugh, \& Fanjaiang, 1975), crearon la prueba para la detección de trastornos cognitivos multifuncional de pacientes geriátricos; enfermedades neuropsiquiatras, estudios clínicos y patologías en este caso demencias. La adaptación al español fue realizada por (Lobo, Saz, \& Marcos, 2002), el instrumento evalúa los trastornos cognitivos a través de 35 preguntas que incluyen la orientación en el espacio, en el tiempo; decodificación, atención, concentración, recuerdo, lenguaje y construcción visual. En la interpretación el punto de corte sugestivo incluye las siguientes mediciones:

29 a 35 discapacidad discreta, déficit ocasionado por una enfermedad depresiva a bajo nivel cultural.

15 - 19 deterioro cognitivo moderado.

5 - 30 deterioro cognitivo normal.

0 - 14 deterioro cognitivo leve.

Otro de los autores es (Ibujés, 2010), presenta como objetivo diagnosticar el nivel de las funciones básicas indispensables en el desarrollo cognitivo, esta prueba permite detectar la situación de las áreas perceptivo motrices; se caracteriza por ser individual en un tiempo libre, puede ser aplicada a un número mayor de 30 niños y niñas, la finalidad es conocer las funciones que favorecen en el adelanto cognoscitivo.

- Beneficia en la identificación de dificultades.

- Incluye consignas para el esquema corporal, dominancia lateral.

- Abarca, el área temporal, la coordinación dinámica.

- Implica el área receptiva sensorial, perceptivo visual, la asociación auditiva y expresiva.

- Incorpora la pronunciación, la memoria por secuencia auditiva; la coordinación visual auditiva motora.

- Contiene la memoria visual, discriminación auditiva, coordinación viso-motora, la atención y fatiga.

El proceso de investigación que el estudio adoptó fue el exploratorio, al describir sobre un tema poco conocido con insuficiente información como es el asesoramiento 
psicopedagógico, las funciones básicas y el déficit cognitivo moderado y severo; además, se fortaleció el conocimiento mediante un registro de búsqueda obtenido de revistas y documentos en español e inglés, entre ellas: Proquest, Scopus, Science Direct; divulgaciones con una antigüedad de 5 años; de esta forma se habitúa y familiariza con la temática al profundizar en los saberes para consolidar las conclusiones.

En el diagnóstico de una persona que presenta déficit cognitivo moderado y severo se aplica el estudio mencionado por (Álvarez, 1994) enfocado en el desarrollo cognoscitivo, siendo esencial aplicar la escala de inteligencia de Wechsler para niños y niñas revisada (WISC-R), que permite medir el coeficiente intelectual (CI) y la evaluación de necesidades. Una vez almacenada la información se procede al análisis, que beneficia en la detección de los puntos clave que conllevan a estimular habilidades cognitivas, teniendo como propósito efectuar el diseño de una propuesta de estrategias y orientaciones dirigidas a la familia y los docentes.

De igual manera se aplicó técnicas cualitativas y cuantitativas que beneficiaron en el diagnóstico, determinación e intervención del déficit cognitivo moderado y severo.

Finalmente se aborda la evaluación del programa de intervención, efectuado de forma continua en el inicio, el desarrollo de cada actividad hasta la última etapa. En este sentido, se resalta que los estudios de Maclure y Davies (1994), sobre capacidad cognitiva postulan que el bajo desempeño, la escasa habilidad para tomar decisiones, las limitaciones en la solución de problemas; aspectos que generan barreras en la socialización, comunicación, argumentación y exteriorización de pensamientos, generando afectaciones en el nivel comportamental, procedimental y actitudinal. Perspectiva posibilita el fortalecimiento de las capacidades, el pensamiento crítico, creativo y lógico, teniendo como fin contribuir en el progreso intelectual.

En la aplicación de la prueba (WISC-R), se obtiene las siguientes puntuaciones

Tabla 1. Puntuaciones prueba (WISC-R),

\begin{tabular}{lll}
\hline \multicolumn{1}{c}{ Indicadores } & Puntuaciones \\
\hline Comprensión Lectora & 69 & 67 \\
\hline Razonamiento Perceptual & 62 \\
\hline Memoria de Trabajo & 70 \\
\hline Velocidad de procesamiento & 60
\end{tabular}

Compilado por: grupo investigador

El Test Neuropsicológico (WISC-R) se orienta a niños y adolescentes (6 años 0 meses y 16 años 11 meses. Según (Perea, 2012) integra los niveles desde la primaria (seis a once años), secundaria (diez a quince) y Bachillerato (hasta 16 años), de esta forma determina las habilidades cognitivas, que contiene las funciones: atención, pensamiento, concentración memoria y lenguaje. 
Según (Vaucheret, y otros, 2017) se relaciona con las habilidades intelectuales generales (comprensión verbal y razón perceptiva) y de procesamiento cognitivo (memoria de trabajo y velocidad), mantienen una vinculación con las teorías de la inteligencia de razonamiento fluido y memoria de trabajo.

Comprensión verbal (CV) según (Aponte \& Zapata, 2015) incluye las habilidades verbales, las relaciones entre conceptos, la definición de vocablos, la agudeza social, el juicio práctico, los conocimientos adquiridos, prontitud e intuición oral. Costa de 5 subpruebas:

Semejanzas (abstracción y generalización a partir de dos conceptos dados)

Vocabulario (conocimiento léxico, precisión conceptual y expresión verbal)

Comprensión (razonamiento solución de problemas cotidianos)

Información (capacidad de obtener, almacenar y recobrar sapiencias adquiridas)

Adivinanzas (habilidades para integrar información)

Razonamiento Perceptivo según (Arrubla, 2020) contiene: las habilidades constructivas, de formación y codificación de concepciones no-verbales, el análisis sensorial y el proceso sincrónico. Costa de 4 subpruebas:

- Cubos: habilidades de análisis, síntesis y organización viso-espacial, en tiempos determinados.

- Conceptos: formación de nociones y categorías mediante material visual.

- Matrices: razonamiento por analogías e integración de información visual.

- Figuras incompletas optativas: Capacidades de reconocimiento y organización perceptible controlada.

Memoria de Trabajo: capacidad de retención, acumulación, codificación, transformación, generación de información. Subpruebas:

- Dígitos: memoria inmediata y de trabajo, incluye la secuenciación, panificación, alerta y flexibilidad cognitiva.

- Letras y números: retención y combinación de información, organización y elaboración según consignas.

- Aritmética: optativa, control de tiempo, analiza las habilidades de razonamiento numérico, velocidad en el manejo y reorganización de la información, atención y memoria a corto plazo.

Velocidad de procesamiento de la información, según (De Vicente, 2017) incluye, la habilidad para focalizar la atención, exploración, ordenación y discriminación visual con rapidez y eficacia. Subpruebas claves:

- La rapidez asociativa, aprendizaje, percepción visual, coordinación viso-manual, atención, motivación y resistencia frente a tareas repetitivas. 
Búsqueda de Símbolos (BS): mide habilidades de rapidez asociativa, aprendizaje, percepción visual, coordinación viso-manual, atención, motivación y resistencia frente a tareas repetitivas).

- Animales (prueba optativa, atención selectiva, y planificación en la búsqueda ordenada versus desordenada de información.

Entre los puntos fuertes sobresale:

- El análisis de las destrezas en los grupos normativos se realiza en función de la edad, o el nivel de aptitud alcanzado.

- Promueve la identificación de debilidades y fortalezas con diferentes posibilidades comparativas de comprensión verbal y razonamiento perceptivo, en diferentes niveles de confianza).

En los puntos débiles

- Existe la crítica a la memoria de trabajo porque sólo incluye tareas auditivas (números, letras y problemas aritméticos); y a la prueba de conceptos al centrarse en la inteligencia general; sin embargo a nivel interpretativo observa las capacidades no verbales.

- Incluye las pruebas de comprensión, historietas y competencia social expresada de forma verbal.

Tabla 2. Prueba WISC - Resultados cuantitativos

\begin{tabular}{|c|c|c|c|c|c|}
\hline \multirow[b]{2}{*}{ Indicadores } & \multicolumn{4}{|c|}{ Puntuaciones típicas } & \multirow[t]{2}{*}{ Total } \\
\hline & $\begin{array}{l}\text { Comprensión } \\
\text { verbal (CV) }\end{array}$ & $\begin{array}{l}\text { Razonamiento } \\
\text { Perceptual }\end{array}$ & $\begin{array}{l}\text { Memoria } \\
\text { de Trabajo }\end{array}$ & $\begin{array}{l}\text { Velocidad de } \\
\text { procesamiento }\end{array}$ & \\
\hline Cubos & & 8 & & & 8 \\
\hline Semejanzas & 5 & & & & 5 \\
\hline Dígitos & & & 2 & & 2 \\
\hline Conceptos & & 4 & & & 4 \\
\hline Claves & & & & 9 & 9 \\
\hline Vocabulario & 4 & & & & 4 \\
\hline Letras y números & & & 5 & & 5 \\
\hline Matrices & & 2 & & & 2 \\
\hline Comprensión & 7 & & & & 5 \\
\hline Total & 14 & 14 & 7 & 9 & 44 \\
\hline
\end{tabular}

Compilado por: grupo investigador

En función del coeficiente intelectual se establece las siguientes puntuaciones: 
- Entre 70 y 50 discapacidad leve (rango en el que se encuentra la mayor parte de población con conflictos intelectuales).

- Entre 50 y 35, la discapacidad sería moderada, existe la necesidad de supervisión y educación especial.

- Entre 35 y 20 discapacidad intelectual grave, son considerados legalmente incapacitados.

- Debajo de 20, discapacidad intelectual profunda, genera daños neurológicos que limitan la actuación e interacción con el medio.

Presentación y conducta: presencia personal apropiada, aseo normal, responsable

Valoración inicial: con sustento en la información obtenida se confirma que las dificultades implican un déficit intelectual de tipo moderado; las funciones cognitivas que deben ser estimuladas son la atención y memoria.

Justificación teórica de las decisiones, las funciones cognitivas a estimular en la estudiante con déficit intelectual son atención y memoria, requiriendo la aplicación de estrategias de trabajo lúdico, pedagógico y estimulación; que tienen como fin mejorar el aprendizaje, el desempeño y rendimiento.

\section{Resultados.}

Según (Picas, y otros, 2007), los orientadores y asesores psicopedagógicos emplean procedimientos que aportan en el bienestar personal y social conllevando a la cooperación activa en la vida escolar. Desde esta visión la calidad educativa se relaciona con el control emocional y las relaciones interpersonales; situación que favorece en el proceso de enseñanza y aprendizaje, de forma particular en la autoestima, percepción y competencia propia; disminuye las barreras en la participación interactiva favoreciendo en la inclusión significativa en el ámbito didáctico, curricular y metodológico.

De acuerdo con (García, Rosales, \& Sánchez, 2003), en la labor educativa la práctica docente incluye un conjunto de acciones que orientan a los estudiantes, maestros, maestras, familias y agentes comunitarios teniendo como fin asesorar a los directivos en aspectos formativos. Desde esta perspectiva los autores (Bello, Naranjo, Cano, \& Sánchez, 2017) mencionan que la asesoría psicopedagógica permite desarrollar habilidades que aporten en la solución de conflictos; según (Lozano, y otros) a través de metodologías enfocadas en la prevención se controla las dificultades de aprendizaje en la diversidad de contextos. El asesoramiento psicopedagógico es necesario diagnosticar las necesidades educativas en sus diferentes niveles siendo necesario:

- Identificar las capacidades y limitaciones en la comunidad pedagógica. 
- Sensibilizar a los maestros directivos y actores del proceso en el rol que desempeñan para la mejora educativa.

- Promover la participación proactiva de todos los integrantes de la comunidad para la gestión de búsqueda de soluciones problemáticas de esta manera se comunican los resultados obtenidos a través de diferentes etapas.

Según, (Figueredo, Campuzano, \& Rodríguez, 2019) el principio de prevención incluye la identificación y cuidado a determinados grupos de riesgo; de esta forma aplica acciones preventivas y actúa sobre circunstancias negativas utilizando el diálogo, la planificación sistémica y el diseño del sistema de apoyo (Lastre \& Jerez, 2019) afirma: con el avance de la ciencia y la tecnología el Ministerio de Educación exige la formación profesional con sustento en competencias, la interdisciplinariedad y el trabajo autónomo; elementos necesarios para alcanzar el desarrollo de las funciones básicas que abarcan la atención, concentración y memoria.

(Pérez, 2008), considera trascendental fortalecer las habilidades intelectuales en niños y niñas que presentan capacidad severa y moderada, el propósito es optimizar la identificación, la observación, la recordación, la clasificación, el análisis y el resumen; aspectos relevantes que contribuyen en el aprendizaje significativo; (Aponte \& Zapata, 2015) de esta forma ayuda en el razonamiento deductivo, el entendimiento, la generalización, la creatividad, el pensamiento estratégico, la toma de decisiones, la atención y la concentración.

Según (Mañas, González, \& Cortés, 2020), el deterioro cognitivo severo hace referencia a la pérdida de memoria, problemas de conducta, movilidad y comunicación; en este sentido el paciente no puede valerse por sí mismo, solicitando el apoyo de otras personas para solventar sus necesidades básicas como alimentación traslación a cualquier sitio caracterizándose por ser vulnerable a infecciones especialmente neumonía, por lo expuesto (Fernández, 2017) manifiesta que es ineludible observar y analizar el lenguaje corporal que incluye los movimientos, la expresión facial, los sentimientos, requiriendo el contacto físico en el control de emociones; además es significativo vigilar los hábitos de higiene y cambios posturales.

\section{Conclusiones.}

- La discapacidad no implica ser incapacitado por esta razón es trascendental recordar que el respeto a los derechos incluye la igualdad en el entorno, (Maclure \& Davies, 1994) mencionan: no es necesario fomentar la compasión o paternalismo, se requiere emplear un trato digno; (Martín \& Rodríguez, 2015) siendo preciso fortalecer la interacción de forma directa, independientemente si se encuentra acompañado; (Portellano, Martínez, \& Zumárraga, 2009) además, no utilizar un tono de voz infantil, el lenguaje es claro y sencillo, que facilite la comprensión; evitar el manejo de frases complejas y largas. 
- Según (Tirapu \& Luna, 2008) la atención a los infantes inicia desde el nacimiento, especialmente en diferentes aspectos como la dificultad para caminar, hablar, aprender, entender normas generales; percibir las consecuencias de sus actos, resolver problemas y pensar con lógica. (Pazmiño, Núñez, López, \& Pazmiño, 2019), afirman que las conductas de adaptación incluyen destrezas de cuidado personal, habilidades sociales y de comunicación, actitudes en el ámbito escolar o laboral, el aprendizaje de rutinas, el pedir ayuda y el uso del dinero.

- Por lo expuesto, en cuanto a los padres se requiere impedir la negación sobre todo cuando se enteran de la discapacidad de su hijo porque se aferran a la idea que el diagnóstico no es correcto, que sus hijos no tienen incapacidad; negándose a cualquier pensamiento que afirma la realidad. El evitar el sentimiento de culpabilidad durante la fase de análisis disminuye la depresión, la baja autoestima que en ocasiones conlleva a la inestabilidad familiar y en otros casos finaliza con la desintegración del hogar.

- Los padres la familia de los niños y niñas o adolescentes con discapacidad deben asumir una posición activa, según (López, Sagñay, Vega, \& Mera, 2019), es prioritario apoyar con diferentes acciones que favorezcan la atención, la comprensión, el lenguaje, la autoconfianza, autovaloración y autoestima. El padre o madre al asistir a la unidad educativa tendrá mayor oportunidad de conocer las dificultades o limitaciones de los hijos. El docente fortalecerá el diálogo y comunicación con los docentes; para mejorar el conocimiento investigará en la web, o consultará a profesionales.

- Es necesario conocer los derechos del ser humano, las leyes vinculadas a la discapacidad de esta forma se logrará establecer relaciones positivas con el docente y el personal de la institución a través del intercambio de información, el desarrollo de planes, programas y adaptaciones en referencia a las necesidades educativas; además los encuentros con otros padres promueven el compartir consejos prácticos y apoyo en el área emocional, así, la paciencia, tolerancia y respeto aporta la superación de dificultades; por las razones expuestas es significativa la motivación familiar durante proceso enseñanza aprendizaje.

En realidad, no existen personas discapacitadas, sólo personas con distintos grados de aptitud"

Henry Viscandi

Dedico este trabajo a Dios, quien siempre ha sido el autor de mi vida y mi destino, a Holguer Darío

Miniguano Yánez y doctora Gabriela Cruz

(Departamento de Bienestar Universitario UTA) 


\section{Referencias bibliográficas.}

Aponte, M., \& Zapata, M. (2015). Caracterización de las funciones cognitivas de un grupo de estudiantes con trastornos específicos del aprendizaje en un colegio de la ciudad de Cali, Colombia Psychologia. Avances de la disciplina 2013, 7 (1). Cali, Colombia : Avances de la disciplina .

Arrubla, R. (3 de Diciembre de 2020). Inducción de procesos cognitivos a partir de referentes semánticos basados en el reconocimiento y priming visual con desenlace trágico. ÁNFORA. Fundación Universitaria del Área Andina, 27(48), 23. Obtenido de https://publicaciones.autonoma.edu.co/index.php/anfora/article/view/668/495

Ausubel, D. (1983). Teoría del aprendizaje significativo. (https://s3.amazonaws.com/academia.edu.documents/38902537/Aprendizaje_signifi cativo.pdf?AWSAccessKeyId=AKIAIWOWYYGZ2Y53UL3A\&Expires $=1553926$ 948\&Signature=yldSZtQnoQ2p1mJih7yQ0EdDC2U\%3D\&response-contentdisposition=inline\%3B\%20filename\%3DTEORIA_DEL_APRENDIZJ,Ed.) Trillas, 1(1), 10.

Bello, M., Naranjo, N., Cano, J., \& Sánchez, D. (5 de Junio de 2017). Perfil Cognitivo y Psicolinguístico y su Relación con la Lectoescritura en un Preescolar con Síndrome de Down. (https://www.aidep.org/sites/default/files/2018-04/RIDEP47-Art9.pdf, Ed.) Revista Iberoamericana de Diagnóstico y Evaluación - e Avaliação Psicológica. RIDEP , 2(47), 16.

De Vicente, M. (3 de Octubre de 2017). Cognifit Salud, Cerebro \& Neurociencia. Recuperado el 20 de Mayo de 2018, de Aprendizaje Cognitivo: Tipos de aprendizaje, una guía educativa: https://blog.cognifit.com/es/aprendizaje-cognitivo-tiposaprendizaje/

Fernández, E. (2017). Desarrollo de la inteligencia emocional a través de la expresión corporal . Madrid, España: Universidad de la Rioja.

Figueredo, E., Campuzano, R., \& Rodríguez, C. (2019). Estrategia compensatoria dirigida a la estimulación del pensamiento en escolares con discapacidad intelectual leve., 6(2). Dilemas Contemporáneos: Educación, Política y Valores, 6(2).

Folstein, M., Folstein, S., McHugh, P., \& Fanjaiang, G. (1975). Examen cognoscitivo mini mental. Madrid, España: Assessment Reources, Odessa, Florida.

García, J., Rosales, J., \& Sánchez, E. (2003). El asesoramiento psicopedagógico como construcción de significados compartidos: un estudio sobre su dificultad. Cultura y Educación, 15(2), 129-148.

García-Cerda, L., Rodríguez-Fernández, O., Betancourt-Galindo, R., Saldívar-Guerrero, R., \& Torres-Torres, M. (2003). Síntesis y propiedades de ferrofluidos de magnetita. Superficies y Vacío., 16(1), 28-31.

Ibujés, J. (2010). Guia de aplicación, evaluación y pautas básicas de recuperación pedagogica para estudiantes de los centros educativos del proyecto. Quito, Ecuador: Ministerio de Educación del Ecuador. 
Instituto Colombiano de Bienestar Familiar (ICBF). (2010). Orientaciones pedagógicas para la atención y la promoción de la inclusión de niñas y niños menores de seis años con discapacidad cognitiva. Bogotá - Colombia: Secretaría Distrital de Integración Social (SISD). Obtenido de https://www.icbf.gov.co/sites/default/files/cartillacognitiva-7.pdf

Lastre, D., \& Jerez, S. (2019). La asesoría psicopedagógica. Su implementación en las instituciones educativas. Revista científica Olimpia, 16(55).

Lobo, A., Saz, P., \& Marcos, G. (2002). Adaptación Mini prueba del estado mental. Madrid, España: ZARADEMIP.

López, M., Sagñay, F., Vega, S., \& Mera, I. (29 de Marzo de 2019). El entorno familiar y el aprendizaje cognitivo. (file:///C:/Users/PC-146/Downloads/420Texto\%20de1\%20art\%C3\%ADculo-1786-4-10-20190423.pdf, Ed.) Revista Indexada El Latindex 2.0. Revista Digital, 3(2), 18. doi:ISSN 2602-8085

Lozano, N., Ruival, P., Riva, S., Mancilla, M., Alvarez, L., Dhers, A., \& Rodriguez, M. (s.f.). Evaluación de las Funciones Ejecutivas de niños entre 6 y 12 años: Normalización de la Batería Neuropsicológica ENFEN en la zona sur de la Provincia de Buenos Aires. Universidad Nacional de Lomas de Zamora (UNLZ), 22(2), 49, 71.

Maclure, S., \& Davies, P. (1994). Aprender a pensar, pensar en aprender. Barcelona, España: Gedisa.

Manzano, S., \& Morocho, J. (2018). Beneficios de la aplicación del Método Montessori para mejorar el aprendizaje de la escritura en los jóvenes con déficit cognitivo leve y moderado en edades comprendidas entre 15 - 25 años. Quito, Ecuador: Universidad Central del Ecuador.

Mañas, M., González, B., \& Cortés, P. (2020). Historias de vida de personas con discapacidad intelectual: Entre el acoso y exclusión en la escuela como moduladores de la identidad. Asociación Investigación, Formación y Desarrollo de Proyectos Educativos, 5(1).

Martín, P., \& Rodríguez, A. (Diciembre de 2015). Capítulo 1. La intervención desde la base neuropsicológica y metodologías que favorecen el rendimiento escolar. Ministerio de Educación de España. Universidad Internacional de La Rioja, 29. Obtenido de file:///C:/Users/PC-146/Downloads/Intervencinconbaseneuropsicolgica.pdf

Pazmiño, J., Núñez, B., López, J., \& Pazmiño, M. (12 de Mayo de 2019). Inteligencia emocional: una competencia para fortalecer el desarrollo cognitivo, el pensamiento crítico, la toma de decisiones y el rendimiento académico . Ciencia Digital, 3(2). doi:http://cienciadigital.org/revistacienciadigital2/index.php/CienciaDigital/article/v iew/472

Perea, V. (1 de 12 de 2012). Los test neuropsicológicos. Componentes negro psicológicos de la conducta y evaluación neuropsicológica. Neuropiscológica , 31. Obtenido de http://www.sld.cu/galerias/pdf/sitios/rehabilitacionlogo/17_test_neuropsicologicos.pdf 
Pérez, E. (2008). Desarrollo de los procesos atencionales . Madrid, España: Universidad Complutence de Madrid. Obtenido de https://eprints.ucm.es/8447/1/T30734.pdf

Picas, J., Cano, M., Lidon, C., Pérez, J., Ballús, E., \& Pera, C. (2007). Manual de asesoramiento psicopedagógico (Vol. 17). Graó. Madrid, España: Graó.

Portellano, J., Martínez, R., \& Zumárraga, L. (2009). Evaluación neuropsicológica de las funciones ejecutivas en niños-entrevista. Universidad Complutense de Madrid, 6.

Rodríguez Yela, P. (2016). Propuesta de un programa de intervención psicopedagógica para trabajar habilidades cognitivas en una estudiante de zona rural con déficit intelectual. Madrid, España: Universidad Internacionacional De La Rioja.

Tirapu, J., \& Luna, P. (2008). Neuropsicología de las funciones ejecutivas. Manual de neuropsicología. Dialnet, 2, 42.

Vásquez, R. (30 de Marzo de 2018). Antecedentes históricos en el desarrollo de la Psicopedagogía en Chile. Pilquen-Sección Psicopedagogía, 15(1), 12. Obtenido de file:///C:/Users/PC-146/Downloads/Dialnet-

AntecedentesHistoricosEnElDesarrolloDeLaPsicopedag-6573068.pdf

Vaucheret, E., Puga, C., Básalo, G., José, M., Pintos, P., Trossero, I., \& Agosta, G. ..-1. (25 de Junio de 2017). Fluencia verbal: un test neuropsicológico breve para la detección de trastornos cognitivos en pediatría. Acta Neurológica Colombiana. Scielo. Acta Neurológica

Colombiana,

6.

Obtenido

de http://www.scielo.org.co/pdf/anco/v33n3/0120-8748-anco-33-03-00142.pdf

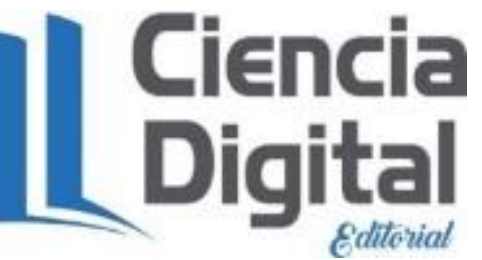




\section{Para citar el artículo indexado}

López Pazmiño , M. N. (2020). Asesoramiento psicopedagógico: propuesta de intervención para reforzar las funciones básicas en una adolescente con déficit cognitivo moderado y severo. AlfaPublicaciones, 3(2), 6-24. https://doi.org/10.33262/ap.v3i2.41

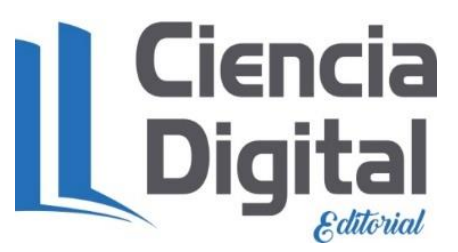

El artículo que se publica es de exclusiva responsabilidad de los autores y no necesariamente reflejan el pensamiento de la Revista Alpha Publicaciones.

El artículo queda en propiedad de la revista y, por tanto, su publicación parcial y/o total en otro medio tiene que ser autorizado por el director de la Revista Alpha Publicaciones.
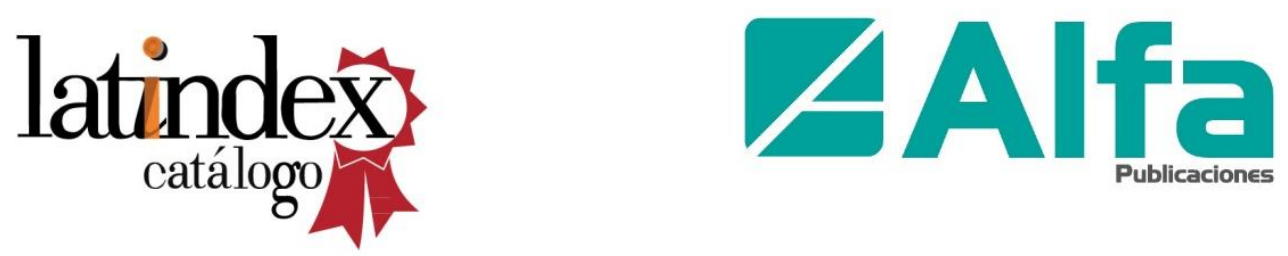